\title{
EL DESARROLLO DE LA PROFESIONALIZACIÓN PEDAGÓGICA, UNA REFLEXIÓN NECESARIA
}

\author{
Maribel Asín Cala, Dra. C. \\ Universidad de Oriente. Santiago \\ de Cuba. Cuba \\ maribela@uo.edu.cu
}

\author{
Daniel Fuentes Almaguer, Dr. C. \\ Universidad de Oriente. Santiago \\ de Cuba. Cuba \\ daniel.fuentes@uo.edu.cu
}

Palabras claves: Profesionalización - profesionalidad docente profesionalidad pedagógica - educación superior - formación inicial y continua.

\section{Keywords: Professionalization - teaching professionalism -} pedagogical professionalism - higher education - formation initial and continual
Recibido: 12 de Octubre de 2017

Aceptado: 06 de Noviembre de 2017

\section{RESUMEN}

En este artículo se reflexiona sobre los principales elementos teóricos necesarios para analizar la profesionalización pedagógica de los claustros de los centros de educación superior, teniendo en cuenta que constituye un imperativo de este nivel en la época contemporánea con el fin de garantizar la formación de un hombre nuevo, capaz de aprender a hacer, aprender a convivir, aprender a ser, de aprender a aprender y aprender a enseñar. Se significa que la profesionalización pedagógica les permitirá a los docentes de las universidades alcanzar el desarrollo de habilidades y competencias necesarias para desarrollar con éxito su labor formadora.

\section{ABSTRACT}

This article reflects on the main theoretical elements necessary to analyze the pedagogical professionalization of the cloisters of higher education centers, taking into account that it constitutes an imperative of this level in the contemporary era in order to ensure the formation of a man new, able to learn to do, learn to live together, learn to be, learn to learn and learn to teach. It means that the pedagogical professionalization will allow the teachers of the universities to achieve the development of skills and competences necessary to successfully develop their training. 


\section{INTRODUCCIÓN}

La época actual se caracteriza por el vertiginoso desarrollo de la ciencia y la técnica, generando un gran caudal de conocimientos, por tanto, se necesita de un hombre capaz y altamente calificado. Al respecto Añorga, J. (1995) expresó: "El avance de la Ciencia y la Tecnología, las transformaciones sociales y los problemas del medio ambiente; han acrecentado en los últimos años, la contradicción dialéctica entre educación y sociedad.

Además, se hace cada vez más evidente el pobre nivel de utilización del potencial humano, justamente cuando las exigencias sociales han aumentado y se requiere un hombre más capaz, más pleno y más humano" (1).

Papel relevante en este proceso lo ocupan los profesores, figura que a través de la historia de la humanidad ha sido altamente valorada por ser la persona que se encarga de manera consciente y sistematizada de transmitir a las nuevas generaciones el acervo históricocultural a tenor de los intereses y necesidades de la época, como premisa para prepararlas para la vida y para asumir una actitud transformadora en el presente y en el futuro.

Fidel Castro Ruz (1981), en el discurso pronunciado en Ciudad Escolar Libertad el 7 de julio expuso ideas sobre el educador que constituyen referentes para comprender la importancia, papel y tareas del profesional de la educación, al expresar que el educador debe ser “ ... un ejemplo de revolucionario, comenzando por el requisito de ser un buen profesor, un trabajador disciplinado, un profesional con espíritu de superación, un luchador incansable contra todo lo mal hecho y un abanderado de la exigencia.

El educador no debe sentirse nunca satisfecho con sus conocimientos. Debe ser un autodidacta que perfeccione permanentemente su método de estudio, de indagación, de investigación. Tiene que ser un entusiasta y dedicado trabajador de la cultura (...) En la medida en que un educador esté mejor preparado, en la medida que demuestre su saber, su dominio de la materia, la solidez de sus conocimientos, así será respetado por sus alumnos y despertará en ellos el interés por el estudio, por la profundización en los conocimientos. Un maestro que imparta clases buenas, siempre promoverá el interés por el estudio en sus alumnos..." (2)

La aspiración es tener maestros y profesores bien preparados en las diferentes esferas de la cultura, la pedagogía, la didáctica, la psicología y otras ciencias afines al arte de educar, instruir, enseñar, desarrollar y formar a las nuevas generaciones y a la sociedad en general, y también identificados con su profesión que están ejerciendo. Sin embargo, en la práctica profesional de los docentes se aprecian insuficiencias pedagógicas, didácticas, psicológicas, culturales, éticas, entre otras, que no les permiten desarrollar un proceso formativo de calidad.

A lo anterior se le agregan las demandas que la sociedad le realiza a los centros de educación superior de garantizar egresados altamente preparados, exigiéndose, por tanto, mayor profesionalización en los docentes. El artículo propone analizar y reflexionar acerca de los elementos teóricos relacionados con la profesionalización pedagógica de los claustros de los centros de educación superior. 


\section{DESARROLLO}

La actividad pedagógica requiere del docente determinadas cualidades positivas de su personalidad, y está demostrado que no hay otra labor que requiera de tantas cualidades positivas para lograr los resultados a que aspira. Autores diversos señalan que las condiciones mínimas u obligaciones fundamentales del maestro se refieren a:

En lo ético: trabajar para que los más altos valores iluminen y dirijan la conciencia y conducta integral de los individuos, hasta elevarlos a categoría de personalidades responsables.

En lo científico: trasmitir los elementos básicos de la ciencia y despertar la inquietud para ampliar el conocimiento.

En lo estético: estimular en las nuevas generaciones la expresión artística y capacitarlas para apreciar la belleza de la naturaleza y las obras de arte, que deben considerarse como medios de elevación y dignificación humana.

En lo filosófico: integrar la formación profesional con una concepción del mundo y del hombre insertado en la realidad histórica, de modo que luego pueda trasmitirla en la escuela. En lo social: el maestro debe convertirse en agente estructurador y perfeccionador de la democracia, a fin de contribuir eficazmente a la rectificación de las injusticias sociales y señalar normas de más justa convivencia.

En lo económico: enseñar el aprovechamiento inteligente de los recursos naturales de su propio país y de los medios de transformación en beneficio de todos los pueblos.

En lo cívico: hacer de sus discípulos ciudadanos que sepan cumplir sus deberes y hacer uso correcto de sus derechos, como miembros de un estado americano que, por sobre todas las cosas, debe garantizar las libertades del hombre y fomentar la cooperación internacional.

El maestro debe tener sólidas convicciones morales que le permitan activar en todos los momentos de su vida en correspondencia con los ideales más progresista y justos del desarrollo social.

\section{Profesionalización docente. Conceptualización.}

En la actualidad la profesionalización constituye uno de los principales retos en las diferentes esferas de la sociedad porque precisamente la sociedad tiene el propósito de brindar servicio de calidad. En tal sentido el sector educacional, en sus diferentes niveles, no escapa a esta exigencia. Lograr tal propósito posibilitará resolver problemas, particularmente los que tienen que ver con la calidad de la enseñanza y la estabilidad de los claustros de docentes, con la calidad y el sentido de pertenencia a la profesión requerida.

El concepto de profesionalización docente está vinculado con otros conceptos como profesión, profesionalidad, profesionalismo, profesional. En ocasiones se confunde y/o relaciona con otros conceptos como autoperfeccionamiento docente, autopreparación profesional, superación profesional, desarrollo profesional, formación profesional, entre otros. Con el propósito de esclarecer dicha relación y puntualizar la definición de profesionalización docente se analizarán brevemente dichos términos. 
Para García Ramis L. (1996) el autoperfecionamiento docente es la actividad autotransformadora que presupone el cambio del docente centrado en el dominio y comprensión profunda de los fines y naturaleza de la actuación profesional, incluyendo los mecanismos que facilitan su cambio sistemático y su autoanálisis.

Más adelante, en esa misma obra, plantea:" autoperfeccionarse es un constante reanálisis de la información sobre los modos de actuar, los procedimientos, las motivaciones, las conceptualizaciones sobre la labor pedagógica, que generan procesos de búsqueda y transformaciones a partir de la propia experiencia y de la experiencia ajena y que recodifica, reorganiza y sistematiza todo el sistema de trabajo del maestro hacia estadios superiores de desarrollo conscientemente determinados" (3)

En diferentes literaturas, olvidando que son categorías pedagógicas diferentes, se utilizan cono sinónimo los conceptos desarrollo profesional y formación profesional.

Así diferentes autores consultados, fundamentalmente los seguidores de la Educación de avanzada, expresan que el desarrollo profesional en un sentido amplio se relaciona con el desarrollo de una persona en su ámbito profesional. Más específicamente, el desarrollo profesional de los profesores, se refiere al crecimiento profesional que alcanza un profesor como resultado de su experiencia y el examen sistemático de su quehacer docente. Incluye entre sus componentes experiencias formales, como la asistencia a actividades de formación específicamente preparadas, congresos, reuniones profesionales, etc. e informales como lecturas de publicaciones profesionales, aprendizajes no planificados, experiencias y vivencias.

Puntualizan que una característica del desarrollo profesional es que es un proceso a largo plazo, donde el docente en formación o post graduado desempeña un papel activo, reflexionando críticamente sobre su actuación y tomando en consideración el conocimiento adquirido con las nuevas experiencias, donde deben incluyan oportunidades y experiencias planificadas que promuevan el crecimiento y el desarrollo en la profesión docente y las escuelas se convierten en verdaderas comunidades de aprendizaje.

Este desarrollo profesional se concibe como un proceso colaborativo que sin suprimir espacio para el trabajo y la reflexión personal, consigue los mayores beneficios en las interacciones significativas no sólo entre profesores, sino entre estos y otros miembros de la comunidad.

Para los autores de este trabajo resultan interesantes los planteamientos de González Maura V. (2004) cuando define el desarrollo profesional como "un proceso permanente, continuo y gradual de tránsito hacia la autodeterminación en el ejercicio de la docencia, que implica necesariamente la reflexión crítica y comprometida del profesor con la transformación de la práctica educativa y la calidad de su desempeño, en un ambiente dialógico y participativo, en el contexto histórico-concreto de su actuación profesional."(4). 
En diferentes artículos se precisan como rasgos fundamentales que definen el contenido del concepto superación:

- La superación es un proceso.

- Tiene un carácter continuo, prolongado, permanente y transcurre durante el desempeño de las funciones directivas, a diferencia de la formación que constituye una etapa inicial, de preparación, en el desarrollo del directivo que puede anteceder al momento de asumirlas.

- Su finalidad es el desarrollo del directivo para su mejoramiento profesional y humano,

- Sus objetivos son de carácter general: ampliar, perfeccionar, actualizar, complementar, conocimientos, habilidades y capacidades, y promover el desarrollo y consolidación de valores.

Esto distingue la superación de la capacitación que tiene un significado más técnico o práctico.

Por su parte Valiente, P. (2001) concibe la profesionalidad como el conjunto de competencias que con una organización y funcionamiento sistémico hacen posible la conjugación armónica entre el "Saber", "Saber hacer" y "Saber ser" en el sujeto, que se manifiesta en la ejecución de sus tareas con gran atención, cuidado, exactitud, rapidez y un alto grado de motivación; que se fundamenta en el empleo de los principios, métodos, formas, tecnologías y medios que corresponden en cada caso, sobre la base de una elevada preparación (incluyendo la experiencia) y que puede ser evaluada a través del desempeño profesional, y en sus resultados.

Vinculado a la profesionalización está el desempeño profesional, vista como la capacidad de un individuo para efectuar acciones, deberes y obligaciones propias de su cargo o funciones profesionales que exige un puesto de trabajo. Esta se expresa en el comportamiento o la conducta real del trabajador en relación con las otras tareas a cumplir durante el ejercicio de su profesión. Este término designa lo que el profesional en realidad hace y no sólo lo que sabe hacer.

Por su parte, la superación profesional es entendida como un conjunto de procesos de enseñanza-aprendizaje que posibilita a los graduados universitarios la adquisición y el perfeccionamiento continuo de los conocimientos y habilidades requeridas para un mejor desempeño de sus responsabilidades y funciones laborales.

Es decir se refiere a las acciones como: la adquisición, actualización y perfeccionamiento, principalmente de conocimientos y habilidades, mejorar el desarrollo de sus responsabilidades profesionales, entre otros, lo cual ha estado presente en las primeras etapas de esta investigación, pero en el caso específico de este período, los docentes, además de las acciones señaladas, deben habilitarse para impartir clases de otras asignaturas para los cuales no han sido preparados en su formación universitaria. 
Lo antes planteado significa, adquirir conocimientos y habilidades nuevas y transferir habilidades pedagógicas y didácticas a situaciones propias de otras asignaturas, que tienen cierta especificidad, y para lo cual la preparación recibida no los capacita.

Por otra parte, la superación es algo que se puede hacer siempre, existan o no necesidades concretas que la determinen, puede habilitar o no a la persona para hacer algo concreto, y en las condiciones actuales, no se trata solo de superación, hay que lograr que los docentes puedan dar clases en estas nuevas circunstancias, o sea, hacerlos aptos para estas nuevas funciones no previstas en su formación inicial. De ahí que se decidió continuar analizando otros términos que permitan un mayor acercamiento a la actividad que debe desarrollarse con los docentes en la situación actual.

Como se puede apreciar los conceptos analizados anteriormente tienen como común denominador los que van dirigidos a:

- Dominio y comprensión profunda de los fines y naturaleza de la actuación profesional

- Reflexión crítica y comprometida del profesor con la transformación de la práctica educativa y la calidad de su desempeño

- Crecimiento profesional

- Proceso colaborativo, dialógico

- Posibilitan al graduado la adquisición y perfeccionamiento continuo de los conocimientos, habilidades básicas y especializadas, así como los valores éticoprofesionales requeridos para un mejor desempeño de sus responsabilidades y funciones como docente con vista a su desarrollo cultural integral.

- Revisión y renovación de conocimientos, actitudes y habilidades previamente adquiridas, determinado por la necesidad de actualizar los conocimientos como consecuencia de los cambios y avances de la tecnología y de las ciencias.

Lo anteriormente expresado y el análisis de la bibliografía consultada sobre profesionalización, permiten comprender la relación con los conceptos anteriormente planteados, su carácter de proceso dinámico, de desarrollo y evolución de una ocupación, más allá de su aceptación como rango y estatus adquirido.

La profesionalidad es una cualidad que puede ser atribuible a una institución o individuo que realiza su trabajo específico con relevante capacidad para cumplir eficientemente su desempeño profesional. Se manifiesta en ejecutar sus tareas con gran atención, cuidado, exactitud, rapidez y competencia. Se fundamenta en emplear los principios, métodos, formas y medios que corresponden a su actividad profesional, basada en una elevada preparación y experiencia.

La profesionalidad se manifiesta en el dominio y empleo de los principios, métodos, formas y medios que corresponda en cada caso, sobre la base de una elevada preparación, incluyendo la experiencia. 
En la Wikipedia se expresa que la profesionalización es un proceso social mediante el cual se mejoran habilidades con el propósito de hacerlas más competitivas en su profesión u oficio, es decir, la profesionalización tiende a establecer normas de conducta, de calificación y cualificación de manera que los miembros de esa profesión actúen en correspondencia con las mismas.

Otro elemento importante a considerar al abordar la profesionalización es lo relacionado con las tendencias que existen para abordar esta temática. En la revisión de la bibliografía relacionada con el tema se aprecia coincidencia al determinar las tendencias de la profesionalización, y se observan tres tendencias. La primera, interpreta la profesionalización como una categoría de máxima generalidad, como proceso y como principio.

\section{Profesionalización como categoría}

La concepción de mayor relevancia y, por ende, la más utilizada como referente por varios investigadores, es la dada por la UNESCO (1993), abogando por una educación que mengüe el desfasaje existente entre la educación que se brinda y las necesidades individuales y sociales. Considerando la profesionalización como el concepto central que debe caracterizar esta nueva etapa educativa y es definida como el desarrollo sistemático de la educación fomentando en la acción y el conocimiento especializado, de manera que las decisiones en cuanto a lo que se aprende, a cómo se lo enseña y a las formas organizativas para que ello ocurra, se tomen de acuerdo a: los avances de los conocimientos científicos y técnicos, los marcos de responsabilidad preestablecidos, los criterios éticos que rigen la profesión y, los diversos contextos y características culturales.

En este sentido, se conciben dos ejes de acciones en la dimensión de la profesionalización dentro de los sistemas educativos. Un eje institucional y otro pedagógico. El eje institucional concibe la profesionalización de los Ministerios de Educación, los niveles intermedios y la escuela. El eje pedagógico incluye la profesionalización del proceso de enseñanza.

Este enfoque engloba todo un proceder educativo en términos gerenciales y didácticos, lo que hace que algunos investigadores y docentes la interpreten como una cualidad de los sistemas educativos. Se considera que esto ha condicionado la no precisión del estatus categorial del término dentro de la Pedagogía Profesional y el conjunto de ciencias pedagógicas, lo que ha conllevado a la no operacionalización o a las variadas formas en que se han realizado.

\section{Como proceso}

La profesionalización vista como proceso, según criterio de los autores consultados, posibilita el diseño de procesos de formación de profesionales, bajo ciertas prácticas, en contextos escolares, comunitarios, y empresariales, dentro de ellos hay quienes acentúan la relación dialéctica entre estos contextos y otros que los contraponen. 
Se comparten los criterios planteados por Añorga, J. Y Addine, F. quien realizan precisiones importantes acerca de la profesionalización docente.

Añorga Morales (1999), asume la profesionalización como un proceso continuo que contiene cuatro niveles: formación vocacional inicial, formación del futuro egresado, formación del recién graduado y la formación continua. Su continuidad, a nuestro juicio, en lo personológico es relativa, en tanto, el proceso de formación y desarrollo de la personalidad en lo profesional, es mucho más dinámico, siendo objeto de innumerables distensiones.

En lo particular, distingue Addine Fátima, (s/f) que la profesionalización debe contribuir a la formación y desarrollo del modo de actuación profesional, desde una sólida comprensión del rol, tareas y funciones, expresada en la caracterización del objeto, la lógica y los métodos de las ciencia, la lógica de la profesión y un contexto histórico determinado.

\section{Como principio}

La mayor cantidad de trabajos que se reportan en la literatura científica consultada están en esta dirección, varias pueden ser las razones, se considera que teniendo como punto de partida la propia sociología de la educación, en lo referente a estructuras de dirección, las políticas y prácticas de centralización y descentralización curricular, unido a la dinámica del trabajo del profesional de la educación, a estos niveles se pueden acometer ciertas empresa investigativas propias de su quehacer cotidiano.

Los autores consultados coinciden en reconocer la necesidad de imprimirles ciertas características en calidad de principio, unos, a los procesos de diseño curricular y, otros, a la ejecución del proceso formativo, es decir asumir la profesionalización como un principio a tener en cuenta en modelación de los diseños curriculares, y que por tanto debe verse reflejado en el perfil del egresado. La profesionalización permite modelar el proceso de formación de profesionales, atendiendo esencialmente al perfil del egresado, posibilitando su materialización en el diseño curricular, en las actividades académicas, laborales e investigativas.

Estos aspectos, dada su amplitud y variedad de criterios puede ser objeto de otra investigación, porque en dependencia del aspecto que se esté analizando puede verse, así por ejemplo en la formación de profesores se plantea que todas las asignaturas y actividades deben propiciar la profesionalidad pedagógica, por tanto, es un principio, pero también se plantea que la misma se desarrolla a lo largo de la carrera y en ejercicio de la profesión, es, por tanto, un proceso.

De forma general, hay consenso respecto a que durante la formación del futuro profesional se deben ofrecer los elementos pedagógicos, psicológico y didácticos que les permitan una vez graduado ejercer la profesión docente y que una vez graduado puede continuar el desarrollo de su profesionalización a través de cursos, postgrados, diplomados, maestrías y doctorados. 
Otros autores consideran la profesionalización no es un evento espontáneo o un estadio de gracia en el que se halla una ocupación, sino que es un proceso continuo, representando el grado en el que los miembros de una ocupación comparten un cuerpo común de conocimiento y utilizan estándares compartidos de práctica en el ejercicio de ese conocimiento y que lejos de asumirla como posesión individual del conocimiento experto y el dominio de habilidades, la práctica profesional del docente es un proceso de acción y reflexión cooperativa, de indagación y experimentación, donde el profesor aprende al enseñar y enseña porque aprende, interviene para facilitar y no imponer ni sustituir la comprensión de los alumnos, y al reflexionar sobre su intervención ejerce y desarrolla su propia comprensión.

Chacón, N. (2004) por su parte considera que la profesionalización docente expresa la síntesis entre los valores y cualidades morales que caracterizan la esencia humanista de la labor del maestro o profesor y el dominio de los contenidos de la enseñanza, de los métodos y de las habilidades profesionales que garantizan el desempeño de su función, avalado por la experiencia práctica acumulada, los resultados y los logros obtenidos en su vida profesional.

Entonces, debe entenderse que la profesionalización docente está vinculada a la calidad de la educación, desarrollo y formación de la sociedad, en su sentido más general, y de los individuos en cada una de las edades, en lo particular y que en la misma inciden diferencias agencias educativas, los contextos de actuación del maestro.

Lo anterior implica comprender que la profesionalización docente no se refiere solo a la formación de profesionales, sino que va más allá y llega a la calidad de la labor que se realiza, traducida en los resultados alcanzados en la educación, instrucción, enseñanza, aprendizaje, formación y desarrollo de la personalidad de los educandos. También es importante considerar el modelo del profesional a que se aspira, los contextos de actuación y el modo de actuación de los mismos.

A juicio de los autores de la presente investigación es pertinente referirse a profesionalidad pedagógica, ya que la labor del profesor de educación superior va más allá del proceso de enseñanza aprendizaje y llega hasta el proceso pedagógico (proceso que engloba al proceso docente educativo y al proceso de enseñanza aprendizaje).

En la bibliografía consultada, de forma general, hay consenso respecto a que el docente graduado lograr la profesionalización a través de cursos, postgrados, diplomados, maestrías y doctorados, encierra expectativa como aspirante y como graduados, dentro de ellas se destacan:

- La profesionalización como preparación y desarrollo profesional: actualización, preparación constante y permanente, una formación especializada, lograr un proceder científico y creativo, enriquecer conocimientos, ampliar expectativas en el área profesional, acercarme a la vanguardia de la labor docente, y poder contar con herramientas necesarias para desempeñarse lo mejor posible. 
- La profesionalización para adquirir mayor eficiencia y desempeño docente: tener mayor preparación para brindar un mejor servicio, y obtener una formación de avanzada en la educación moderna, ser parte importante de las innovaciones, siendo capaces de afrontar los problemas presentes y futuros en materia de educación, tener mayor acervo cultural para ofrecer una educación de calidad, detectar, superar y proponer soluciones rápidas y adecuadas a las problemáticas que diariamente se presentan en el quehacer educativo, elevar la calidad del proceso de enseñanza aprendizaje y docente educativo.

- La profesionalización para mejorar y transformar la práctica docente: comprender y transformar las prácticas educativas, fortalecer y renovar la práctica educativa, ser capaces de plantear alternativas de solución a problemáticas de la labor docente, adecuándose a los cambios que se promueven en educación, a partir de la reflexión crítica de la práctica docente, romper con el trabajo rutinario y ser creativos e innovadores.

- La profesionalización como actualización académica profesional: lograr la actualización constante, para el mejoramiento sistemático de nuestra labor educativa y la posibilidad de apropiarse de elementos metodológicos, científicos, pedagógicos, psicológicos, filosóficos, sociológicos, didácticos que permitan la crítica, el análisis y la realización de aportes, oportunidad de conocer diferentes teorías y tendencias actuales que sustentan nuestra labor docente, oportunidad de enriquecer conocimientos, ampliar expectativas en el área profesional y acercarse a la vanguardia de la labor docente, haciendo uso de la teoría.

Se asume el criterio de Castro Alegret, Pedro Luis y otros (2015) cuando afirman que la profesionalidad pedagógica, expresa la integración de la ideología, los valores y cualidades morales que caracterizan la esencia humanista de la profesión, con los conocimientos pedagógicos, culturales y científico - tecnológicos necesarios para la labor educativa del docente, fraguados y avalados en el trabajo pedagógico, en la experiencia práctica acumulada, los resultados y logros obtenidos en su desempeño y modos de actuación, como concreción y desarrollo de sus competencias profesionales.

Se precisan las siguientes dimensiones de la profesionalidad pedagógica:

1) Conciencia de los deberes pedagógicos: implica el compromiso social de la educación de las nuevas generaciones en las condiciones de la época, a partir del protagonismo como estilo de trabajo pedagógico y como modos de actuación de los estudiantes, lo que presupone una educación en y para la democracia del socialismo cubano, concibiendo como parte del proceso formativo los escenarios de las transformaciones del modelo económico cubano y la lucha ideológica que libra nuestro pueblo por mantener su independencia y soberanía nacional.

2) Concepción pedagógica más integradora, teniendo en cuenta el enfoque axiológico, para el tratamiento adecuado de la instrucción y de la educación, atendiendo a los componentes cognitivo, afectivo - volitivo, ideológico y actitudinal, así como la relación de la ciencia y tecnología con los valores y la ideología, como parte de una 
cultura humanista, en la enseñanza aprendizaje (intencionalidad ideológica), dominio del contenido y empleo adecuado de la metodología para enseñar y educar.

3) Enfoques pedagógicos multidisciplinar e interdisciplinar de los conocimientos en la enseñanza y aprendizaje, para comprender problemas complejos de la ciencia, la tecnología, el medio ambiente y el mundo globalizado en que vivimos, lo que repercute en nuevos modos de concebir, relacionarse y de actuación en el trabajo pedagógico.

4) La utilización adecuada de los medios y recursos tecnológicos de que se disponen para la labor educativa, los que no sustituyen el papel de la influencia educativa del docente con sus estudiantes, la familia y la comunidad.

5) Desarrollo de una Ética de la cooperación profesional, que supere la barrera de los celos profesionales, de "pocos amigos" o de "cada maestro con su librito", que impiden, una comunicación franca e intercambio de ideas y experiencias profesionales con fluidez, receptividad y respeto, con el aporte de los diferentes enfoques a la solución de problemas comunes, sin subvalorar las posibilidades que tiene "el otro" para ello, la tolerancia, la amplitud y flexibilidad de pensamiento, entre otros, para practicar la ayuda mutua y la solidaridad en el trabajo.

Estos retos se transforman en las dimensiones de la profesionalidad pedagógica del profesor universitario, entendidas como los planos del contenido del trabajo pedagógico, expresado en sus componentes internos y la correlación de estos componentes con las necesidades sociales, expresadas en formas de exigencias del desarrollo, la práctica o encargo social.

Diversos autores destacan a la profesionalización como aspecto importante en la conformación de la identidad profesional pedagógica ya que la identidad profesional es el resultado de la formación, constituida por el conjunto de competencias adquiridas, como por el posicionamiento social de la profesión docente y sus instituciones de formación pedagógica, y la construcción de una identidad profesional que asuma las exigencias de los tiempos actuales, vale decir, demostrar capacidad de diagnóstico, manejo flexible y creativo de los instrumentos técnicos, comunicativos o intelectuales disponibles, ser capaz de atender los emergentes y la diversidad, a la par de poseer capacidades afectivas y relacionales.

Por tanto, la profesionalización conlleva la adquisición y desarrollo de las características, capacidades, competencias y habilidades de la profesión docente. Se relaciona con la cultura profesional ya que se necesita mejorar las tareas y las relaciones laborales debido a que la profesionalización requiere del contexto social para validarse y reconocerse.

El gran desafío que tiene hoy la educación de producir saltos cualitativos en los diferentes procesos donde los docentes y estudiantes figuran como protagonistas, ha llevado al fortalecimiento de los estudios relacionados con su desempeño y sus formas de evaluación.

Los que se han planteado como fin fortalecer la calidad de la educación desde diferentes aspectos sociales teniendo en cuenta que el ejercicio profesional del docente es una necesidad para las pretensiones del sistema educacional. 
Sobre esta base diferentes investigadores han abordado algunos rasgos esenciales de un docente, reflexionando sobre cuáles deben ser sus habilidades didácticas para que se promueva un aprendizaje de calidad, cuáles son sus misiones y tareas, cuál es su responsabilidad para que se materialicen las expectativas educacionales de la sociedad, cuál debe ser su modelo de desempeño, cómo evaluar su desempeño, cómo propiciar su creatividad profesional, entre otras.

Se considera además que la evaluación en educación no puede ser considerada como un proceso aislado, sino en sistema con los demás componentes de un todo integrado, por lo que no debemos verla como un resultado, y mucho menos como algo acabado, es necesario ver a la evaluación en movimiento, en correspondencia con el objeto a evaluar que extraemos para analizarlo e interpretarlo, pero que al hacerlo, éste puede ir sufriendo cambios sistemáticos simultáneos, en este caso por la intervención del hombre.

El resultado responde al objetivo en relación con la satisfacción del problema, o sea un resultado efectivo y de calidad; en el proceso de formación de los profesionales el impacto estará dado en la calidad de sus egresados que será la medida de cómo enfrentan y resuelven los problemas profesionales y las necesidades de la sociedad que originaron el problema.

En el empeño de lograr la profesionalización pedagógica de los claustros de los centros de educación superior, no deben olvidarse las siguientes condiciones:

- La existencia de políticas y estrategias nacionales e institucionales dirigidas a tal fin.

- Interrelación (articulación e integración) entre los centros formadores, instituciones, organismos y empresas (centros donde realizan la práctica pre profesional) y los organismos empleadores para dar, desde la formación inicial hasta la permanente, una respuesta que privilegie desde el pregrado el carácter vivencial de la profesión.

- Condiciones laborales: salario, estímulos, condiciones materiales del puesto de trabajo, clima comunicacional y organizacional, facilidades para la superación, etc.

- Calidad de la formación y superación inicial y permanente lo que implica:

- Ingreso a los centros formadores (calidad del ingreso-cualidades, conocimientos, actitudes, aptitudes, rasgos y características de la personalidad-, motivación, orientación profesional, compromiso,),

- Profesionalización de las actividades y tareas docentes, variedad, contextualización (currículo, permanente y postgraduado),

- Correspondencia con sus necesidades y potencialidades,

- Concepción y aplicación de un sistema de formación permanente que abarque desde la formación inicial hasta la formación científica que favorezca el tránsito continuo, sistémico y sistemático de los docentes,

- Tutoría y/o acompañamiento,

- Evaluación de la calidad de la educación y del desempeño profesional.

- Gestión institucional de los diferentes procesos formativos. 
- Reconocimiento social de la labor profesional pedagógica y enaltecimiento de la profesión.

- Generar y privilegiar el conocimiento profesional colaborativo, crítico que favorezca la interpretación, comprensión y reflexión sobre la práctica pedagógica y su transformación.

Los elementos abordados permitirán cumplir con las exigencias de la UNESCO, referidas a la necesidad de elevar la calidad de la enseñanza y por consiguiente la exigencia de lograr la calidad de los docentes que laboran en la Educación Superior. En particular resultan importantes lo planteado por Delors, J (1996) en el informe de la Comisión Internacional sobre educación para el siglo XXI presentado en la UNESCO, en su capítulo dedicado al personal docente cuando plantea que nunca se insistirá demasiado en la importancia de la calidad de la enseñanza y, por ende, del profesorado.

Así pues, mejorar la calidad y la motivación de los docentes debe ser una prioridad en todos los países. La importancia de esta temática también fue refrendado en la Conferencia Mundial sobre la Educación Superior de la UNESCO (1998), con la aprobación de documentos que sustentan la necesidad de la educación permanente del profesorado universitario y su formación pedagógica. En uno de estos documentos se especifica: Un elemento esencial para las instituciones de enseñanza superior es una enérgica política de formación del personal.

Se insiste en que deben tomarse medidas adecuadas en materia de investigación, así como de actualización y mejora de sus competencias pedagógicas mediante programas adecuados de formación del personal, que estimulen la innovación permanente, a fin de garantizar la excelencia de la investigación y la enseñanza.

Atendiendo a todo lo anterior se hace necesario que en los centros de educación superior se elaboren estrategias que, considerando los contenidos necesarios para garantizar, a partir de la valoración de cada una de las dimensiones declaradas y las condiciones necesarias, la profesionalización pedagógica de sus claustros.

\section{CONCLUSIÓN}

La sociedad actual demanda una universidad cada vez más desarrollada, con un profesor culto, poseedor de métodos para renovar constantemente sus conocimientos, capaz de planificar, ejecutar y evaluar el proceso de enseñanza- aprendizaje de manera científica y con cualidades que le permitan orientar a sus alumnos y a las personas que influyen en la educación de este, teniendo en cuenta las necesidades sociales, A lo anterior se le agregan las demandas de la sociedad de egresados de las diferentes carreras y especialidades altamente preparados, lo que demanda el desarrollo de la profesionalidad pedagógica de los claustros universitarios. 


\section{BIBLIOGRAFÍA}

1. AÑORGA, J. (1995): La Educación Avanzada: "Una teoría para el mejoramiento profesional y humano": Boletín del CENESEDA. Instituto Superior Pedagógico Enrique José Varona. La Habana. P. 4

2. CASTRO RUZ, FIDEL. Discurso en la graduación del Destacamento Pedagógico Manuel Ascunce Domenechll. 7 de julio de 1981, en Ciudad Escolar Libertad. Periódico Granma. Tercera edición, 9 de julio de 1981. Año 17, No. 161, p.2

3. GARCíA RAMIS, L., ALBERTO VALLE y MIGUEL A. FERRER (1996). Autoperfeccionamiento docente y creatividad, Editorial Pueblo y Educación, La Habana, Cuba. P 19

4. GONZÁLEZ MAURA, VIVIANA. (2002) ¿Qué significa ser un profesional competente? Revista Iberoamericana de Educación p.3

5. CHACÓN ARTEAGA, NANCY (2004). Ética y educación en tiempos de globalización desde la perspectiva cubana. En Formación del profesorado en Cuba. En formato digital.p.33

6. CASTRO ALEGRET, PEDRO LUIS, NANCY CHACÓN ARTEAGA Y LISSETTE MENDOZA PORTALES. (2013). La escuela y la formación de valores. Enfoques y experiencias. Curso Prevento. Pedagogía. 2013. En CD. Pedagogía 2013, Evento Internacional. p 43-44

7. DELORS, J. (1996). La educación encierra un tesoro. Informe a la UNESCO.p.167. Extraído el 18 de octubre de 2015 del sitio www.unesco.org/education/pdf/DELORS_S.PDF

8. VALIENTE SANDÓ, PEDRO. (2001) Concepción sistémica de la superación de los directores de secundaria básica. Tesis presentada en opción al Grado Científico de Doctor en Ciencias Pedagógicas. Holguín 